\title{
Preoperative Mechanical Bowel Evacuation Reduces Intraoperative Bleeding and Operation Time in Spinal Surgery
}

\author{
Majid Rezvani ${ }^{1}$, Reza Abbasi ${ }^{1}$, Homayoon Tabesh ${ }^{1}$, Leila Dehghani ${ }^{2}$, Shahaboddin Dolatkhah ${ }^{3}$, \\ Maryam Nasri ${ }^{4}$, Mohsen Kolahdouzan ${ }^{5}$, Rokhsareh Meamar ${ }^{6,7}$ \\ ${ }^{I}$ Department of Neurosurgery, School of Medicine, Isfahan University of Medical Sciences, Isfahan, Iran \\ ${ }^{2}$ Department of Tissue Engineering and Regenerative Medicine, School of Advanced Technologies in Medicine, \\ Shahid Beheshti University of Medical Sciences, Tehran, Iran \\ ${ }^{3}$ Faculty of Medicine, Isfahan University of Medical Sciences, Isfahan, Iran \\ ${ }^{4}$ BENevis Grovemead Health Centre, London, UK \\ ${ }^{5}$ Department of Surgery, Faculty of Medicine, Isfahan University of Medical Sciences, Isfahan, Iran \\ ${ }^{6}$ Isfahan Endocrine and Metabolism Research Center, Isfahan University of Medical Sciences, Isfahan, Iran \\ ${ }^{7}$ Clinical Research Development Center, Najafabad Branch, Islamic Azad University, Najafabad, Isfahan, Iran
}

\section{Study Design: Randomized clinical trial.}

Purpose: In this study, we evaluated the effect of mechanical evacuation of the bowels prior to operation on intraoperative bleeding. Overview of Literature: Bleeding is the most significant complication in patients undergoing spinal surgery.

Methods: We randomly divided 108 individuals planned to undergo spinal surgery into two age-, sex-, and co-morbidity (especially preoperative hemoglobin [Hb])-matched groups of 54 . The treatment group was administered polyethylene glycol (PEG) before the operation, whereas the control group was not. The exact amount $(\mathrm{mL})$ of bleeding during operation, operative time, and approximate amount of blood transfused were recorded. The volume of bleeding and $\mathrm{Hb}$ level were also recorded 24 and 48 hours postoperatively. Results: $T$-tests revealed that intraoperative bleeding, the volume of transfusion, and operative time were significantly lower in the treatment group than in the control group. Statistically significant correlations of intraoperative bleeding with age, body mass index (BMI), preoperative $\mathrm{Hb}$ levels, operative time, the volume of transfusion, hospitalization time, and 24- and 48-hour postoperative bleeding were observed ( $p=0.001$, all). Repeated measures analysis of covariance after adjusting the covariate variables revealed that the volume of bleeding showed a near-significant trend in the treatment group compared with that in the control group ( $p=0.056)$. Diabetic females had the highest bleeding amount between the groups ( $p=0.03)$. Bleeding was higher in patients with higher $\mathrm{BMI}(p=0.02)$ and was related to operative time $(p=0.001)$ in both the groups.

Conclusions: Preoperative gastrointestinal tract evacuation by PEG administration can decrease intraoperative bleeding in spinal surgeries; however, more research is imperative regarding PEG administration in surgical procedures for this purpose.

Keywords: Polyethylene glycol; Intraoperative complications; Decompression surgery; Surgical blood loss

\footnotetext{
Received Aug 17, 2017; Revised Oct 14, 2017; Accepted Nov 1, 2017

Corresponding author: Rokhsareh Meamar

Isfahan Endocrine and Metabolism Research Center, Isfahan University of Medical Sciences, Isfahan, Iran

Clinical Research Development Center, Najafabad Branch, Islamic Azad University, Najafabad, Isfahan, Iran

Tel: +98-31-33359933, Fax: +98-31-33373733, E-mail: Meamar@pharm.mui.ac.ir
} 


\section{Introduction}

The number of spinal surgeries aiming to correct major spinal deformities or degenerative changes has been increasing. The main cause of this increase is considered to be the increases in the life expectancy as well as the rate of ageing (the number of individuals $>65$ years of age) of the general population [1]. Patients planning to undergo spinal surgeries, such as spinal fusion, experience complications, such as a delay in discharge from hospital, perioperative blood loss, and need for repeat surgery, particularly when they are overweight $[2,3]$. The estimated intraoperative blood loss in spinal fusion surgeries is $907 \pm 775 \mathrm{~mL}$ for posterior spinal fusion, $323 \pm 171 \mathrm{~mL}$ for anterior spinal fusion, and $1,277 \pm 825 \mathrm{~mL}$ for combined procedures [4], which illustrates the fact that there is usually massive bleeding in such surgeries.

Minimally invasive spinal surgeries have long-term outcomes similar to those of open spinal procedures $[5,6]$. There are fewer short-term complications in minimally invasive surgeries than in open procedures, such as decreased need for blood transfusion during surgery, decreased pain postoperatively, shorter hospitalization time, and decreased volume of intraoperative blood loss $[5,7,8]$. Nevertheless, blood loss as a complication of spinal surgeries is a concern in both types of procedures. Spinal epidural hematoma may also occur in patients undergoing multilevel lumbar interventions or in those with coagulopathy disorders [9]. Blood transfusion is required to replace intraoperative blood loss, but there are several transfusion-related morbidities, including acute lung injury and the transmission of infections. High financial costs and mortality are also possible consequences. These complications limit the routine use of autologous transfusion [10-16]. The importance of reserving blood in any medical procedure is evident, and several blood-sparing techniques, including the administration of epidural blockade, desmopressin, aprotinin, tranexamic acid, epsilon-aminocaproic acid, estrogen, bone wax, fibrin sealants, hemostatic sponges, blood-saving techniques, acute hemodilution, and planned autologous transfusion, are recommended to decrease intraoperative bleeding or the need for transfusion $[17,18]$.

The vertebral venous plexus is a large valve-less network of vertebral veins that regulates venous drainage of the spinal cord [19], and similar to other venous systems throughout the body, external pressure can affect its blood flow [20,21]. In our case, the environmental pressure is intra-abdominal pressure, and full bowels can increase intra-abdominal pressure. In the present study, we evaluated whether lowering intra-abdominal pressure via the mechanical evacuation of the gastro-intestinal tract could lower abdominal venous pressure and consequently decrease perioperative bleeding.

\section{Materials and Methods}

This randomized clinical trial study (NCT02493894) was conducted from February 2012 until June 2014 at the neurosurgery ward of Alzahra Hospital, one of the teaching hospitals of the Isfahan University of Medical Sciences. In this study, 108 patients who were admitted to our hospital and underwent spinal surgery (fusion and instrumentation) with a pedicular screw because of spinal column instability, degenerative changes, and canal stenosis were included. The indication for surgery was determined by an experienced neurosurgeon, and the decision for surgery was based on clinical findings, imaging data, and guidelines and reference sources for neurosurgery. Patients with a history of trauma, malignancy, previous spinal surgery, coagulation disorders, active infectious disease, gastrointestinal problems, or severe movement disorders were excluded from the study. Patients receiving anticoagulants or anti-platelet drugs or any medication affecting coagulation were not included. In patients receiving such medications, the surgery was postponed until the medication was discontinued and its effects were diminished. The patients exhibited normal bowel habits during 6 months prior to the surgery.

During this prospective study, the patients were randomly divided into two groups: treatment group $(\mathrm{n}=54)$ and control group ( $n=54)$ (Fig. 1). The patients in both the groups were matched for age; sex; and current morbidity, including diabetes and hypertension; anemia; and preoperative hemoglobin $(\mathrm{Hb})$ levels. Written informed consent regarding the therapeutic plan and the probable side effects was obtained from all patients in the treatment group. The ethics committee of the Isfahan University of Medical Sciences approved the protocol for this study (IRB approval no., 393618), and the study was conducted in accordance with the principles of the Declaration of Helsinki.

The patients' characteristics, including body mass index (BMI), previous medical history (diabetes and hypertension), and $\mathrm{Hb}$ level were recorded. In the treatment group, 


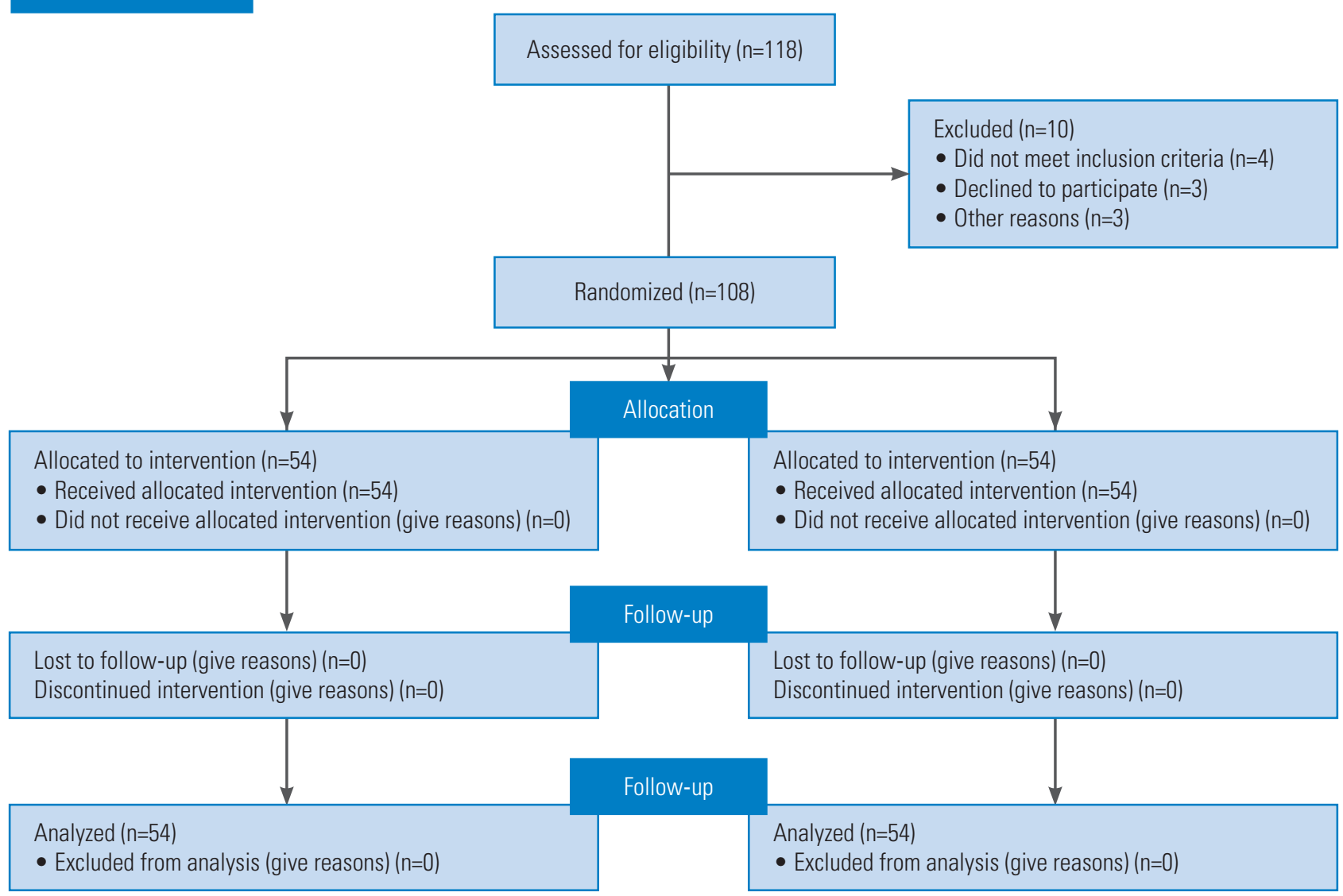

Fig. 1. Consort 2010 flow diagram.

the patients received a liquid diet for 24 hours, followed by polyethylene glycol (PEG) solution $(80 \mathrm{~g} / \mathrm{L})$ every 8 hours for 24 hours. After 48 hours, the bowels were mechanically prepared for the procedure. The operation was performed with the patients in the prone position on a standard surgery table. In all patients, laminectomy was performed, but a facetectomy was also required in $10 \%$ of the patients. None of the patients required posterior lumbar interbody fusion or transforaminal lumbar interbody fusion. All patients received general anesthesia in a similar manner. In the postoperative anesthesia care unit (PACU), vital signs (blood pressure, pulse, respiration, pulse oximetry, and adequate answering) were monitored every 15 minutes by a consultant. Patients were discharged from the PACU when vital signs were normalized.

In both the groups, the operative time and volume of bleeding (in $\mathrm{cm}^{3}$ ) were precisely recorded by the surgical and anesthetics team. The need for blood transfusion during surgery was determined on the basis of preoperative $\mathrm{Hb}$ level and the volume of bleeding during surgery and was calculated according to anesthesiology and surgical standards using the following formula [22]:

$\left\{\frac{\text { Target hematocrit-patient hematocrit }}{\text { Target hematocrit }}\right\} \times 70 \times$ body weight

Immediately following the surgery, the exact rate of bleeding during the operation (in $\mathrm{mL}$ ), operative time, approximate transfusion rate, and fusion level were recorded. The maximum level of fusion performed was 4. The volume of bleeding in the hemovac drain was recorded 24 hours and 48 hours postoperatively. The patients' $\mathrm{Hb}$ levels were measured in 24- and 48-hour intervals after the operation.

After ambulating the patients, they were discharged from the hospital. The duration before the patients were mobilized and the total duration of hospitalization were recorded. The successful outcome of the operation was evaluated on the basis of the patients' clinical conditions and using imaging modalities: computed tomography scans and X-ray radiographs. 
Table1. Characteristics of patients who underwent spinal surgery

\begin{tabular}{|c|c|c|c|c|}
\hline Characteristic & Treatment group & Control group & Total & $p$-value \\
\hline Sex & & & & 0.147 \\
\hline Male & 20 (37) & $27(50)$ & $47(43.9)$ & \\
\hline Female & $34(63)$ & $27(50)$ & $61(56.1)$ & \\
\hline Total & $54(100)$ & $54(100)$ & $108(100)$ & \\
\hline Age (yr) & $55.39 \pm 12.67$ & $57.09 \pm 11.85$ & $56.18 \pm 12.2$ & 0.474 \\
\hline Body mass index $\left(\mathrm{kg} / \mathrm{m}^{2}\right)$ & $24.68 \pm 21.10$ & $24.59 \pm 2.80$ & $24.58 \pm 2.51$ & 0.845 \\
\hline Diabetes & $12(22)$ & $15(27)$ & $27(25)$ & 0.502 \\
\hline Hypertension & $14(26)$ & $16(28)$ & $30(28)$ & 0.665 \\
\hline Preoperative Hb & $13.08 \pm 0.84$ & $13.16 \pm 1.05$ & $13.1 \pm 0.96$ & 0.666 \\
\hline Postoperative $\mathrm{Hb}$ day 1 & $11.7 \pm 0.99$ & $11.56 \pm 1.11$ & $11.6 \pm 1.05$ & 0.490 \\
\hline Postoperative Hb $48 \mathrm{hr}$ & $11.7 \pm 0.95$ & $11.37 \pm 1.0$ & $11.5 \pm 1$ & 0.086 \\
\hline Need for transfusion (mL) & $370 \pm 0.652$ & $870 \pm 1.19$ & & 0.008 \\
\hline No. of fusion levels & & & & 1.000 \\
\hline 2 & $16(29.6)$ & $16(29.6)$ & $32(29.6)$ & \\
\hline 3 & $27(50.0)$ & $27(50.0)$ & $54(50.0)$ & \\
\hline 4 & $11(20.4)$ & $11(20.4)$ & $22(20.4)$ & \\
\hline Operative time (hr) & $2.49 \pm 0.62$ & $2.79 \pm 0.67$ & $2.6 \pm 0.66$ & 0.016 \\
\hline Intraoperative bleeding (mL) & $784.63 \pm 291.5$ & $921.39 \pm 472.99$ & $849.77 \pm 396.62$ & 0.043 \\
\hline Postoperative bleeding $24 \mathrm{hr}(\mathrm{mL})$ & $98.15 \pm 45.64$ & $110.93 \pm 56.08$ & $104.05 \pm 51.05$ & 0.197 \\
\hline Postoperative bleeding $48 \mathrm{hr}(\mathrm{mL})$ & $119.25 \pm 74.57$ & $139.35 \pm 102.28$ & $129.12 \pm 89.40$ & 0.249 \\
\hline Hospitalization time before mobilization (day) & $1.70 \pm 0.571$ & $1.70 \pm 0.633$ & $1.70 \pm 0.60$ & 1.000 \\
\hline Postoperative hospitalization time (day) & $3.63 \pm 1.170$ & $3.87 \pm 1.214$ & $3.73 \pm 1.19$ & 0.296 \\
\hline
\end{tabular}

Values are presented as number (\%) or mean \pm standard deviation. $\mathrm{Hb}$, hemoglobin.

\section{Statistical analysis}

The data were analyzed in the IBM SPSS software ver. 20.0 (IBM Corp., Armonk, NY, USA), using chi-square test and independent $t$-test. Repeated measures analysis of covariance (ANCOVA) and Pearson's correlation tests were used to determine confounder variables that could influence perioperative bleeding in three stages (during surgery, 24 hours after surgery, and 48 hours after surgery). Adjustment for multiple comparisons was performed using Bonferroni test. Repeated measures ANCOVA was used after adjusting confounding variables. All $p<0.05$ were considered as indicating a statistically significant difference.

\section{Results}

In this study, a total of 108 patients who were candidates for the fusion and instrumentation of the lumbar spine, were divided into two groups with 54 patients each. The rates of mortality and morbidity (surgical and anesthetic complications), evaluated at discharge and a week after discharge, were zero. $T$-tests revealed less intraoperative bleeding, less surgical transfusion amount, and reduced operation time in the treatment group than in the control group (Table 1). Regardless of the allocated group, Pearson's correlation tests revealed associations among intraoperative bleeding and the following variables: age $(p=0.001), \mathrm{BMI}(p<0.001)$, preoperative $\mathrm{Hb}(p=0.001)$, operative time $(p<0.001)$, transfusion amount $(p=0.000)$, hospitalization time $(p<0.001)$, and postoperative $24-$ and 48 -hour bleeding $(p<0.001)$. Furthermore, the mobilization of patients prior to discharge was significantly correlated with hospitalization time $(p=0.000)$.

After adjusting covariate variables, including age, sex, $\mathrm{BMI}$, presence of diabetes, preoperative $\mathrm{Hb}$, operative 
time, and transfusion amount, the repeated measures ANCOVA revealed that bleeding in the three stages was significantly different $(p<0.01)$ and that the volume of bleeding showed a near-significant trend in the treatment group compared with that in the control group $(p=0.056)$. In addition, the diabetic females showed the highest bleeding rate in both the groups $(p=0.03)$, and the bleeding rate was higher in the patients with higher BMI $(p=0.02)$. The operative time was longer in the patients in both the groups $(p=0.001)$.

\section{Discussion}

Intra-abdominal pressure is affected by the abdominal content. Hypothetically, decreasing the intra-abdominal contents should decrease the intra-abdominal pressure. Pressure on the abdominal contents would be transmitted to the inferior vena cava and then to the epidural venous system, which could cause increased bleeding during operation [20]. Releasing pressure from the intraabdominal venous plexus should, therefore, improve flow in the venous system surrounding the spine because of the close network with the Batson's plexus. In this study, the possible effect of mechanical gastrointestinal evacuation on surgical bleeding during lumbar spine fusion and instrumentation surgeries was evaluated for the first time. In univariate analysis, intraoperative bleeding and operative time were significantly higher in the treatment group than in the control group; however, in the repeated measures ANCOVA, a near-significant trend was observed. Although a study by Glickman et al. [23] has evaluated the efficacy of the regional usage of synthetic PEG in anastomoses sites to reduce local bleeding, our extensive search of the literature yielded no study similar to ours.

Recent research studies have been conducted on the beneficial aspects of PEG as a tissue-compatible reagent for the prevention of adhesion after laminectomy [24] and as a carrier of regeneration-prompting molecules in spinal cord injuries [25]. The oral administration of PEG in patients suffering from spinal injuries has also been noted in the setting of bowel preparation for colonoscopy [26] and the treatment of neurological bowel dysfunction after spinal cord injuries [27]. Our results support the administration of PEG for bowel evacuation to decrease the volume of intraoperative bleeding during spinal surgeries and to decrease the need for blood transfusion. A study by Stokes et al. [15] $\mathrm{n}$ bleeding-related complications in a group of surgical patients undergoing various surgeries (thoracic, vascular, hip and knee replacement, general, reproductive organ, and spinal surgeries) has demonstrated that the highest cost per hospitalization was associated with bleeding-related complications of spinal surgeries.

Excessive blood loss is defined as a loss of more than one volume of the total blood or $60 \mathrm{~mL} / \mathrm{kg}$ in adults in a period of less than one day [28]. A study by Stokes et al. [15] has shown that $14.3 \%$ of patients who underwent spinal surgery required blood product transfusions and $0.3 \%$ required interventions to control bleeding. A study by Thompson et al. [29] on patients who underwent posterior spinal instrumentation and fusion has revealed that excessive blood loss was statistically associated with the number of levels fused, operative time, and the number of screws. They have also found that the number of levels fused during spinal surgery was the strongest predictive factor for intraoperative blood loss. Similar, our data indicates that higher levels of fusion and longer operative time were associated with further blood loss in spinal surgeries. Other factors influencing the risk of intraoperative bleeding could be older age, obesity, $\mathrm{Hb}$ levels before operation, and the presence of diabetes and hypertension. Similar data from another study has demonstrated that age, a history of prior surgery, or the presence of underlying disease (diabetes and chronic obstructive pulmonary disease) were associated with a higher risk of intraoperative bleeding [15]. Interestingly, we noted that diabetic women had the highest risk of massive blood loss during spinal surgery, which should be considered by surgeons when managing this group of patients. Zheng et al. [30] have reported that among patients who underwent spinal surgeries, the operative time was associated with the number of levels fused, the diagnosis of degenerative scoliosis, and being overweight. Similarly, the study of Zheng et al. [30] has also illustrated that higher preoperative $\mathrm{Hb}$ levels, body weight, and the number of levels fused were the major risk factors predictive of intraoperative bleeding. Patients who experienced more bleeding during the operation experienced more bleeding episodes 24 and 48 hours after the surgery.

\section{Conclusions}

Our statistical analysis illustrated that preoperative gastrointestinal tract evacuation by PEG administration can decrease intraoperative bleeding in spinal surgeries as well 
as reduce operative time and the need for blood transfusion. Older age, obesity, operative time, and underlying morbidity were also associated with higher intraoperative bleeding. Our data demonstrate that patients whose gastrointestinal tracts were evacuated before surgery required less transfusion than patients in the control group. Therefore, we conclude that PEG usage could be a means to reduce blood transfusion rates. However, more evidence is imperative to substantiate our findings that PEG reduces blood transfusion rates in spinal or other surgical procedures.

\section{Conflict of Interest}

No potential conflict of interest relevant to this article was reported.

\section{References}

1. Martin BI, Deyo RA, Mirza SK, et al. Expenditures and health status among adults with back and neck problems. JAMA 2008;299:656-64.

2. Goldstein CL, Macwan K, Sundararajan K, Rampersaud YR. Comparative outcomes of minimally invasive surgery for posterior lumbar fusion: a systematic review. Clin Orthop Relat Res 2014;472:1727-37.

3. Lau D, Khan A, Terman SW, Yee T, La Marca F, Park P. Comparison of perioperative outcomes following open versus minimally invasive transforaminal lumbar interbody fusion in obese patients. Neurosurg Focus 2013;35:E10.

4. Ialenti MN, Lonner BS, Verma K, Dean L, Valdevit A, Errico T. Predicting operative blood loss during spinal fusion for adolescent idiopathic scoliosis. J Pediatr Orthop 2013;33:372-6.

5. Lau D, Lee JG, Han SJ, Lu DC, Chou D. Complications and perioperative factors associated with learning the technique of minimally invasive transforaminal lumbar interbody fusion (TLIF). J Clin Neurosci 2011;18:624-7.

6. Park P, Foley KT. Minimally invasive transforaminal lumbar interbody fusion with reduction of spondylolisthesis: technique and outcomes after a minimum of 2 years' follow-up. Neurosurg Focus 2008;25:E16.

7. Peng CW, Yue WM, Poh SY, Yeo W, Tan SB. Clini$\mathrm{cal}$ and radiological outcomes of minimally invasive versus open transforaminal lumbar interbody fusion.
Spine (Phila Pa 1976) 2009;34:1385-9.

8. Schizas C, Tzinieris N, Tsiridis E, Kosmopoulos V. Minimally invasive versus open transforaminal lumbar interbody fusion: evaluating initial experience. Int Orthop 2009;33:1683-8.

9. Kou J, Fischgrund J, Biddinger A, Herkowitz H. Risk factors for spinal epidural hematoma after spinal surgery. Spine (Phila Pa 1976) 2002;27:1670-3.

10. Engoren M, Mitchell E, Perring P, Sferra J. The effect of erythrocyte blood transfusions on survival after surgery for hip fracture. J Trauma 2008;65:1411-5.

11. Robb WJ. Massive transfusion in trauma. AACN Clin Issues 1999;10:69-84.

12. Rogers MA, Blumberg N, Saint S, Langa KM, Nallamothu BK. Hospital variation in transfusion and infection after cardiac surgery: a cohort study. BMC Med 2009;7:37.

13. Shander A. Financial and clinical outcomes associated with surgical bleeding complications. Surgery 2007;142(4 Suppl):S20-5.

14. Spiess BD. Blood transfusion: the silent epidemic. Ann Thorac Surg 2001;72:S1832-7.

15. Stokes ME, Ye X, Shah M, et al. Impact of bleedingrelated complications and/or blood product transfusions on hospital costs in inpatient surgical patients. BMC Health Serv Res 2011;11:135.

16. Taylor RW, O'Brien J, Trottier SJ, et al. Red blood cell transfusions and nosocomial infections in critically ill patients. Crit Care Med 2006;34:2302-8.

17. Szpalski M, Gunzburg R, Sztern B. An overview of blood-sparing techniques used in spine surgery during the perioperative period. Eur Spine J 2004;13 Suppl 1:S18-27.

18. Wong J, El Beheiry H, Rampersaud YR, et al. Tranexamic acid reduces perioperative blood loss in adult patients having spinal fusion surgery. Anesth Analg 2008;107:1479-86.

19. Nathoo N, Caris EC, Wiener JA, Mendel E. History of the vertebral venous plexus and the significant contributions of Breschet and Batson. Neurosurgery 2011;69:1007-14.

20. Batson OV. The function of the vertebral veins and their role in the spread of metastases. Ann Surg 1940;112:138-49.

21. Park CK. The effect of patient positioning on intraabdominal pressure and blood loss in spinal surgery. Anesth Analg 2000;91:552-7. 
22. Miller RD. Miller's anesthesia: volume 1. 7th ed. Philadelphia (PA): Churchill Livingstone; 2010.

23. Glickman M, Gheissari A, Money S, Martin J, Ballard JL; CoSeal Multicenter Vascular Surgery Study Group. A polymeric sealant inhibits anastomotic suture hole bleeding more rapidly than gelfoam/thrombin: results of a randomized controlled trial. Arch Surg 2002;137:326-31.

24. Wang Y, Liang M, Zheng Z, et al. Adhesion prevention after laminectomy using silk-polyethylene glycol hydrogels. Adv Healthc Mater 2015 Aug 24 [Epub]. https://doi.org/10.1002/adhm.201500392.

25. Papastefanaki F, Jakovcevski I, Poulia N, et al. Intraspinal delivery of polyethylene glycol-coated gold nanoparticles promotes functional recovery after spinal cord injury. Mol Ther 2015;23:993-1002.

26. Korsten MA, Spungen AM, Radulovic M, et al. Neostigmine administered with MoviPrep improves bowel preparation for elective colonoscopy in patients with spinal cord injury: a randomized study. J Clin Gastroenterol 2015;49:751-6.
27. Yi Z, Jie C, Wenyi Z, Bin X, Hongzhu J. Comparison of efficacies of vegetable oil based and polyethylene glycol based bisacodyl suppositories in treating patients with neurogenic bowel dysfunction after spinal cord injury: a meta-analysis. Turk J Gastroenterol 2014;25:488-92.

28. Elgafy H, Bransford RJ, McGuire RA, Dettori JR, Fischer D. Blood loss in major spine surgery: are there effective measures to decrease massive hemorrhage in major spine fusion surgery? Spine (Phila Pa 1976) 2010;35(9 Suppl):S47-56.

29. Thompson ME, Kohring JM, McFann K, McNair B, Hansen JK, Miller NH. Predicting excessive hemorrhage in adolescent idiopathic scoliosis patients undergoing posterior spinal instrumentation and fusion. Spine J 2014;14:1392-8.

30. Zheng F, Cammisa FP Jr, Sandhu HS, Girardi FP, Khan SN. Factors predicting hospital stay, operative time, blood loss, and transfusion in patients undergoing revision posterior lumbar spine decompression, fusion, and segmental instrumentation. Spine (Phila Pa 1976) 2002;27:818-24. 